\title{
Comment on Solberg and Jensenius: The Temporal Dynamics of Embodied Pleasure in Music
}

\author{
MARIA A. G. WITEK[1] \\ Center for Music in the Brain, Dept. of Clinical Medicine, Aarhus University \& Royal Academy of Music, \\ Aarhus/Aalborg \\ PETER VUUST \\ Center for Music in the Brain, Dept. of Clinical Medicine, Aarhus University \& Royal Academy of Music, \\ Aarhus/Aalborg
}

\begin{abstract}
In the paper 'Pleasurable and Intersubjective Embodied Experiences of Electronic Dance Music', Ragnhild Torvanger Solberg and Alexander Refsum Jensenius report on a study in which the movements and self-reported affective responses of a group of dancing participants were recorded and related to structural properties of Electronic Dance Music. They observed that, compared with tracks that had a relatively flat dynamic development, tracks which included a 'break-down', 'build-up' and 'drop' of textural layers were associated with greater changes in movement amount and higher ratings of pleasure. Here I comment on their results and methodological approach and use the opportunity to address the continuous pleasure that was treated as a control in this experiment, discussing some reasons why affective responses to music with more evenly distributed dynamic progressions are so often ignored.
\end{abstract}

Submitted 2016 March 23; accepted 2016 October 23.

KEYWORDS: electronic dance music, pleasure, embodiment, process

THE paper 'Pleasurable and Intersubjective Embodied Experiences of Electronic Dance Music' reports on a study in which the authors Ragnhild Torvanger Solberg and Alexander Refsum Jensenius recorded hand- and head-movements and self-reported intersubjective affective and embodied experiences in response to four Electronic Dance Music (EDM) tracks, with a group of dancing participants in a motion-capture lab lit and decorated to feel like a club.

Due to problems with marker occlusion, the movements of individual participants could not be extracted and the resulting analyses were thus based on overall movement amount of the group. While this inevitably introduced a certain loss on statistical robustness, their results seem to convincingly indicate that there is a clear relationship between certain dynamic structures in EDM and quantity of motion (QoM). By combining plots of the tracks' amplitude with the averaged QoM, the reader can clearly see that, as hypothesized, dynamically deviating points in the music are associated with changes in the participants' movements. The dynamically deviating points of interest to the authors are the 'break-down', 'build-up' and 'drop' of textural layers that are highly characteristic of EDM. The plots, alongside mean and standard deviations of QoM, show that movement decreases during the 'break-down', gradually increases during the 'build-up' and reaches a peak after the 'drop'. These systematic fluctuations in movement are not observed during tracks that don't include this 'break routine' but rather have a more stable dynamic development. Here, the QoM is relatively constant, with a slight gradual decrease over the course of the given track. The paper is the first to document peak pleasurable experiences as reflected in body-movements during dance.

From the web-based questionnaires, which were completed by participants after the recording session, the authors report the percentages of responses corresponding to four degrees of pleasure experienced in response to the tracks ('not pleasurable', 'slightly pleasurable', 'quite pleasurable', 'very pleasurable'). While, as the authors point out, these percentages do indicate that the tracks involving the 'break routine' were experienced as more pleasurable, testing the significance of these differences would have made their interpretations more reliable. Since the authors obtained 4-point Likert scale responses from 15 out of their 
16 participants, we see no practical reason why significance-testing had to be rejected. For example, by first averaging responses across the tracks with versus without the 'break routine' and then comparing ratings to these two conditions with a simple pair-wise t-test, the extent to which the 'break routine' increases experiences of pleasure with EDM could have been determined more precisely.

\section{PEAK PLEASURE EXPERIENCES WITH MUSIC}

Researchers interested in musical pleasure have almost exclusively focused on such momentarily intense emotional arousal in response to peaks in the musical structure, and as Solberg and Jensenius point out in their brief yet comprehensive review of the literature, the theory of expectation is most often used to explain such responses. According to this theory, most famously argued by Leonard B. Meyer (1956), musical structures that in creative ways violate or fulfill our expectations elicit pleasure, or positively valenced emotional arousal. As David Huron (2006) later added, expectation is at the heart of the biological purpose of pleasure: successful predictions will always be rewarded with pleasure in the brain, since being able to predict future events is essential for adaptation and survival. This line of thinking is deeply rooted in a causal approach to reward pursued in the cognitive sciences, where a specific stimulus triggers a series of successive reactions, leading to the pleasure feeling. This series of reactions has been referred to as 'the pleasure cycle' (see Figure 1), and consists of delineated 'wanting', 'liking' and 'learning' components (Berridge \& Kringelbach, 2011; Gebauer, Kringelbach, \& Vuust, 2012).

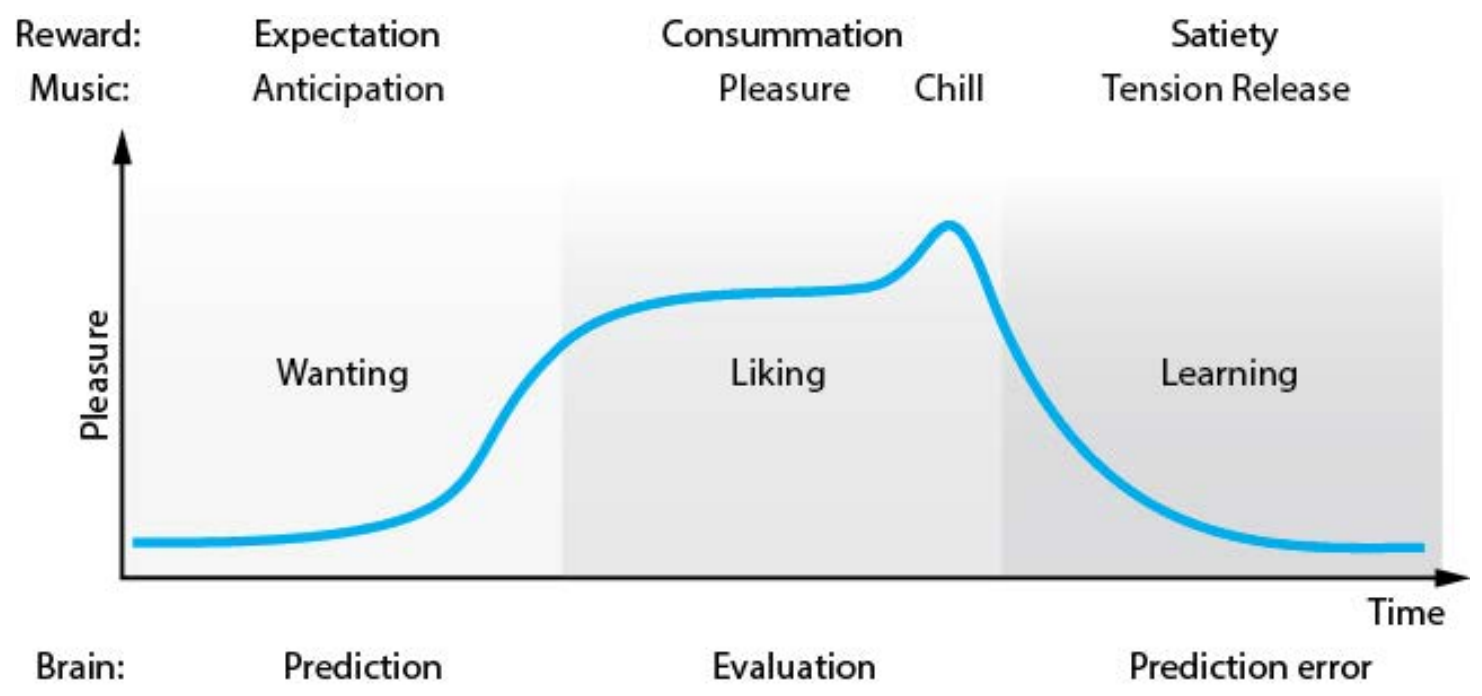

Fig. 1. Reward and musical pleasure cycles, dissociating dynamic components of 'wanting', 'liking', 'chill' and 'learning' during experiences of reward, according to psychological (top panel) and neural processes (bottom panel). Adapted with permission from Berridge and Kringelbach (2011).

\section{CONTINUOUS PLEASURE WITH MUSIC}

While such linear views of pleasure fit nicely with teleologically composed music, such as that involving the kinds of structural peaks seen in EDM tracks with 'break routines', they fall short of explaining the pleasure associated with a wide variety of music that is more repetitive and dynamically flatter. In fact, two of the tracks in Solberg and Jensenius' paper fall into this category. Responses to these tracks were used as controls against which responses to the tracks with 'break routines' could be compared. The authors acknowledge that there is a kind of continuous pleasure associated with such music, and that it has been attributed to the perception-action loop resulting from synchronizing body-movements to a regular beat. In particular, research into musical groove has begun to address the relationship between rhythmic structures, sensorimotor synchronization and pleasure (Janata, Tomic, \& Haberman, 2012; Witek, Clarke, Wallentin, Kringelbach, \& Vuust, 2014; Witek et al., 2016). However, there is a lack of understanding of how the perception-action 
interaction underlying synchronized movements to rhythmic and repetitive music relates to pleasure. While music psychology research, exemplified by the target paper, has made important progress in studying musical pleasure in the form of peak experiences, the focus on such exceptional musical moments has overshadowed the affective significance of music without such large-scale peaks.

One likely reason for why peak pleasure responses have been more often studied than continuous flat pleasure responses is that they stand out. They can be clearly identified and delineated in physiological recordings (Guhn, Hamm, \& Zentner, 2007) neuroimaging (Salimpoor, Benovoy, Larcher, Dagher, \& Zatorre, 2011) and motion-capture (target article). The pleasure we experience during a repeated pattern, such as in groove, is more distributed and thus a given moment of pleasure cannot be demarcated. The 'wanting', 'liking' and 'learning' components of the pleasure experience are not linear, but are mixed and simultaneous (see Figure 2). Unsurprisingly, many have assumed based on this that experiences with music involving large-scale peaks are more intensely pleasurable than experiences with music without peaks. The rating data reported in the paper by Solberg and Jensenius support this assumption, although as mentioned, without reporting the statistical significance of their results. Accepting quantity of motion as an index of pleasure, as the authors do in their paper, it would also be interesting to see whether there were significant differences in overall QoM between the tracks with and without peaks (the authors only report average QoM for the tracks without peaks, and for the 'beat routine' subsections of the tracks with peaks). Because the tracks with peaks also involve significant preceding troughs, i.e. decreases in movement during the 'breakdown', it could be that the overall QoM in fact averages out and is no greater for tracks with than without peaks. A study using a larger set of stimuli could potentially answer this question. But even if such a study showed that indeed music with large-scale peaks is more pleasurable than music without peaks, the albeit lesser pleasure of music without peaks should not be ignored.

\section{Groove: Anticipation/Pleasure/Body-Movement}

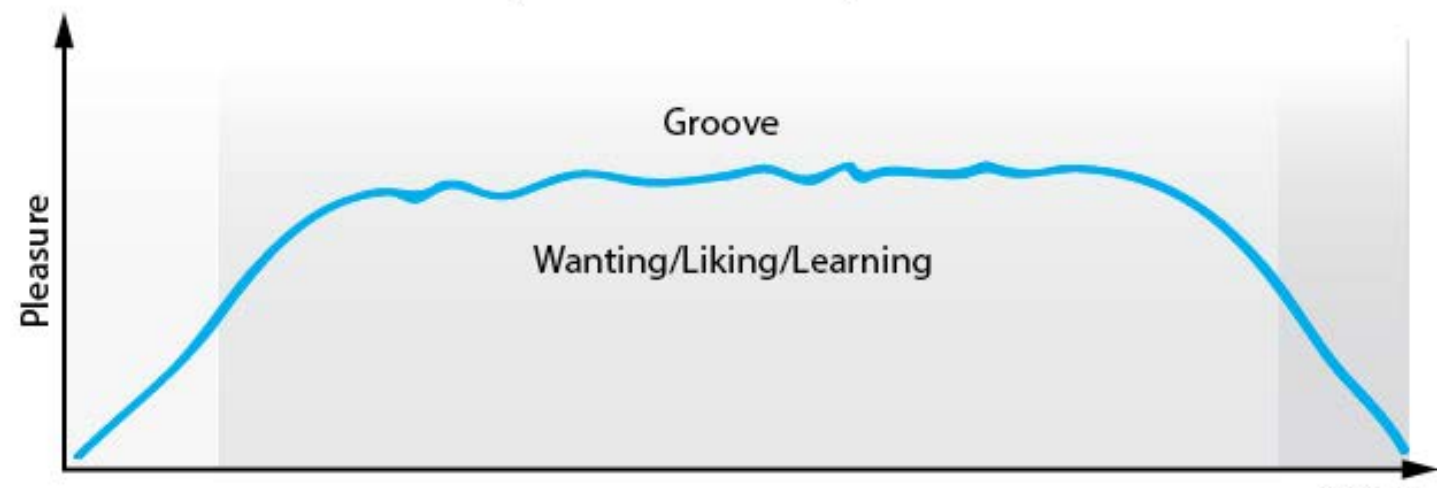

Time

\section{Brain: Prediction/Evaluation/Prediction Error}

Fig. 2. Groove pleasure process, in which 'wanting', 'liking' and 'learning' phases are distributed across the psychological experience of anticipation, pleasure and body-movement (top panel) and at the neural level of prediction, evaluation and prediction error (bottom panel).

Another potential reason for why continuous pleasure without large-scale peaks has been overlooked is that, unlike peak-oriented music, it is not easily explained by an available mechanistic theory like the expectation theory. Elsewhere, one of the authors of the present commentary has argued that in groove, the constant syncopation and cross-rhythmic tendencies of textural layers invite active participation from listeners and dancers, by offering the opportunity to fill in, with their bodies and their minds, the structural gaps revealed by the syncopations (Witek, 2016). In other words, the movements we make when dancing to groove are active contributions to the musical structure. House, techno and other forms of electronic dance music are very good examples of music that affords such embodied filling in, since they overwhelmingly feature and forefront syncopated layers. While Witek accepts that syncopations - which are in fact defined by their function in violating rhythmic expectations - may be stimulating expectations at a micro-level, she rejects that expectation theory can explain the pleasure we experience with groove as a 
whole, since syncopations in groove are constantly repeated. Instead, she considers perspectives in which pleasure is defined as a process or practice rather than a causal result triggered by a specific stimulus (Stephan, Walter, \& Wilutzky, 2013). When viewed as practice, pleasure becomes distributed across body, mind and environment, and its cultural, historical and situated nature can more easily be integrated with its psychological and biological properties (Scheer, 2012). Pleasure, and emotions more broadly, are neither 'inside' humans nor 'outside' in the environment, but emerge in the active engagement of humans with their environments (Wetherell, 2014). Via the related theory of Extended Mind (Clark, 2008; Clark \& Chalmers, 1998), the pleasure of groove is thus understood as radically embodied, or enacted, and rather than groove structure being a stimulus that triggers an affective response, groove affords a more distributed affective experience where - as long as we are moving to the beat - pleasure is maintained. Here, pleasure is not caused by moving - rather, pleasure is to move.

\section{CALLING FOR A NEW MODEL}

While the phenomenological analysis in Witek (2016) provides a first step towards understanding the continuous pleasure we experience with repetitive music without large-scale peaks, a comprehensive theory is needed that also addresses the underlying cognitive mechanisms of such experiences, one that can stand as an alternative to the expectation theory. Establishing the affective significance of the action-perception loop mentioned by Solberg and Jensenius (Overy \& Molnar-Szakacs, 2009), as well as musical entrainment specifically (Trost et al., 2014; Trost, Labbé, \& Grandjean, 2017), could be fruitful points of departure. The authors also mention the importance of dopamine in both reward- and motor-networks of the brain (Keitz, Martin-Soelch, \& Leenders, 2003), and the extent to which body-movement and pleasure are directly related due to this shared neurotransmitter is unknown. Adults with Parkinson's Disease - who not only suffer from severe motor deficits due to dopaminergic dysfunction but also exhibit reward dysfunction, such as depression (Remy, Doder, Lees, Turjanski, \& Brooks, 2005) - may be an interesting population to study for this purpose. In fact, the potential benefit of using groove music in therapeutic interventions for improving gait in Parkinsonianizm has already been addressed (Nombela, Hughes, Owen, \& Grahn, 2013).

\section{SUMMARY}

In this commentary, we have considered the results presented in the paper 'Pleasurable and Intersubjective Embodied Experiences of Electronic Dance Music' by Ragnhild Torvanger Solberg and Alexander Refsum Jensenius. Using motion-capture, they show for the first time that peak pleasure experiences, which are the most commonly studied forms of pleasure in music, can not only be observed in physiological and neural responses during contemplative listening, but also in body-movements during dancing to electronic dance music, specifically in response to the 'break routine'. This turn to embodied affective responses is important in recognizing the active basis of pleasure. However, we point to the potential for learning even more about active forms of musical pleasure by considering more continuous and non-peak oriented kinds of musical pleasure. While more difficult to measure, due to their temporally distributed rather than linearly differentiated cognitive subcomponents, studying pleasurable engagements with repetitive music, such as groove, could establish more forcefully the fundamentally embodied and enactive nature of human affective process. We call for a comprehensive theory of continuous and embodied pleasure in music which can offer alternatives to the expectation theory according to which peak pleasure experiences have been addressed, and suggest that pleasure should here not be treated in a stimulus-response kind of way, but rather as a distributed process between mind, body and music. An important aspect of the target article was not addressed, namely the intersubjective nature of the pleasure experienced with EDM, but we suspect that a theory in which musical pleasure is treated as practice rather than response will allow for an understanding of the experience of electronic dance music that is also socially distributed.

\section{Acknowledgements}

Center for Music in the Brain is funded by the Danish National Research Foundation (DNRF117). 


\section{NOTES}

[1] Correspondence can be addressed to: Dr. Maria A. G. Witek, Center for Music in the Brain, Dept. of Clinical Medicine, Aarhus University Hospital, Noerrebrogade 44, Aarhus 8000, Denmark, maria.witek@clin.au.dk.

\section{REFERENCES}

Berridge, K. C., \& Kringelbach, M. L. (2011). Building a neuroscience of pleasure and well-being. Psychology of Well-Being, 1(1), 1-26. https://doi.org/10.1186/2211-1522-1-3

Clark, A. (2008). Supersizing the mind: Embodiment, action, and cognitive extension. New York: Oxford University Press. https://doi.org/10.1093/acprof:oso/9780195333213.001.0001

Clark, A., \& Chalmers, D. (1998). The extended mind. Analysis, 58(1), 7-19. https://doi.org/10.1093/analys/58.1.7

Gebauer, L., Kringelbach, M. L., \& Vuust, P. (2012). Ever-changing cycles of musical pleasure: The role of dopamine and anticipation. Psychomusicology, 22(2), 152-167. https://doi.org/10.1037/a0031126

Guhn, M., Hamm, A., \& Zentner, M. (2007). Physiological and musico-acoustic correlates of the chill response. Music Perception, 24(5), 473-483. https://doi.org/10.1525/mp.2007.24.5.473

Huron, D. (2006). Sweet anticipation: Music and the psychology of expectation. Cambridge, MA: The MIT Press.

Janata, P., Tomic, S. T., \& Haberman, J. M. (2012). Sensorimotor coupling in music and the psychology of the groove. Journal of Experimental Psychology: General, 141(1), 54-75. https://doi.org/10.1037/a0024208

Keitz, M., Martin-Soelch, C., \& Leenders, K. L. (2003). Reward Processing in the Brain: A Prerequisite for Movement Preparation. Neural Plasticity, 10(1-2), 121-128. https://doi.org/10.1155/NP.2003.121

Meyer, L. B. (1956). Emotion and meaning in music. Chicago and London: University of Chicago Press.

Nombela, C., Hughes, L. E., Owen, A. M., \& Grahn, J. A. (2013). Into the groove: Can rhythm influence Parkinson's disease? Neuroscience and Biobehavioral Reviews, 37(10, Part 2), 2564-2570.

https://doi.org/10.1016/j.neubiorev.2013.08.003

Overy, K., \& Molnar-Szakacs, I. (2009). Being together in time: Musical experience and the mirror neuron system. Music Perception, 26(5), 489-504. https://doi.org/10.1525/mp.2009.26.5.489

Remy, P., Doder, M., Lees, A., Turjanski, N., \& Brooks, D. (2005). Depression in Parkinson's disease: loss of dopamine and noradrenaline innervation in the limbic system. Brain, 128(6), 1314-1322. https://doi.org/10.1093/brain/awh445

Salimpoor, V. N., Benovoy, M., Larcher, K., Dagher, A., \& Zatorre, R. J. (2011). Anatomically distinct dopamine release during anticipation and experience of peak emotion to music. Nature Neuroscience, 14(2), 257-262. https://doi.org/10.1038/nn.2726

Scheer, M. (2012). Are emotions a kind of practice (and is that what makes them have a history)? A Bourdieuian approach to understanding emotion. History and Theory, 51(2), 193-220.

https://doi.org/10.1111/j.1468-2303.2012.00621.x

Stephan, A., Walter, S., \& Wilutzky, W. (2013). Emotions beyond brain and body. Philosophical Psychology, 27(1), 65-81. https://doi.org/10.1080/09515089.2013.828376 
Trost, W., Frühholz, S., Schön, D., Labbé, C., Pichon, S., Grandjean, D., \& Vuilleumier, P. (2014). Getting the beat: Entrainment of brain activity by musical rhythm and pleasantness. Neuroimage, 103(0), 55-64. https://doi.org/10.1016/j.neuroimage.2014.09.009

Trost, W., Labbé, C., \& Grandjean, D. (2017). Rhythmic entrainment as a musical affect induction mechanism. Neuropsychologia, 26, 96-110. https://doi.org/10.1016/j.neuropsychologia.2017.01.004

Wetherell, M. (2014). Trends in the turn to affect: A social psychological critique. Body \& Society, 1-28. https://doi.org/10.1177/1357034X14539020

Witek, M. A. G. (2016). Filling in: Syncopation, pleasure and distributed embodiment in groove. Music Analysis. https://doi.org/10.1111/musa.12082

Witek, M. A. G., Clarke, E. F., Wallentin, M., Kringelbach, M. L., \& Vuust, P. (2014). Syncopation, bodymovement and pleasure in groove music. PloS one, 9(4), e94446.

https://doi.org/10.1371/journal.pone.0094446

Witek, M. A. G., Popescu, T., Clarke, E. F., Hansen, M., Konvalinka, I., Kringelbach, M. L., \& Vuust, P. (2016). Syncopation affects free body-movement in musical groove. Experimental Brain Research. https://doi.org/10.1007/s00221-016-4855-6 